\title{
Comunicación
}

\section{Interrupción de gestación en perras de cría mediante cloprostenol: reporte de casos}

\author{
Gestation termination in breeding bitches by cloprostenol: case report
}

Alfonso Sánchez Riquelme ${ }^{1,2}$, Francisco Arias Ruiz ${ }^{1}$

\section{Resumen}

Se describe el uso de un protocolo abreviado para la interrupción de gestación en perras, mediante la administración subcutánea de cloprostenol en 4 dosis de $1 \mu \mathrm{g} / \mathrm{kg}$ cada 12 horas y 4 dosis de $2 \mu \mathrm{g} / \mathrm{kg}$ cada 12 horas a 10 perras de 2-5 años, con gestaciones de 25-30 días. El diagnóstico de gestación, la estimación de edad gestacional y la confirmación de la interrupción de gestación se realizaron mediante exámenes ecográficos. Los resultados indicaron que la interrupción de gestación se observó como reabsorción, posterior a la sexta administración en cinco hembras con gestaciones entre 25 y 27 días y como aborto en las otras cinco hembras con gestaciones entre 28 y 30 días posterior a la séptima administración de cloprostenol. En todos los casos se observó presencia de efectos colaterales transitorios como salivación, jadeo y vómito. Se concluyó que el tratamiento resultó eficaz y seguro.

Palabras clave: perra; interrupción de gestación; cloprostenol

\section{AbstraCT}

This paper describes the use of an abbreviated protocol for the interruption of gestation in bitches by subcutaneous administration of cloprostenol in 4 doses of $1 \mu \mathrm{g} / \mathrm{kg}$ every 12 hours and 4 doses of $2 \mu \mathrm{g} / \mathrm{kg}$ every 12 hours. The bitches were from 2 to 5 years old, with gestations between 25 and 30 days. The diagnosis of gestation, the estimation of gestational age and the confirmation of the interruption of gestation were carried out

\footnotetext{
${ }^{1}$ Facultad de Medicina Veterinaria y Agronomía, Escuela de Medicina Veterinaria, Universidad de Las Américas, Viña del Mar, Chile

${ }^{2}$ E-mail: asanchez@udla.cl/www.udla.cl

Recibido: 17 de enero de 2018

Aceptado para publicación: 14 de junio de 2018
} 
through sonographic examinations. The pregnancy interruption was observed as reabsorption after the sixth administration in five females with gestations between 25 and 27 days, and as an abortion in the other five females with gestations between 28 and 30 days after the seventh administration of cloprostenol. In all cases, the presence of temporary side effects such as salivation, panting and vomiting was observed. It was concluded that the treatment was effective and safe.

Key words: bitch; gestation termination; cloprostenol

\section{INTRODUCCIÓN}

La interrupción de gestación en perras de valor reproductivo es un motivo de consulta frecuente en la clínica de pequeños animales (De La Sota et al., 2002; Fontbonne, 2010). Esta situación, por lo general, obedece a apareamientos no deseados o no programados por los criadores o propietarios. Actualmente, como criterio ético-científico, se considera fundamental el diagnóstico de preñez previo a cualquier tratamiento; es decir, realizar un procedimiento basado en la evidencia y no ciego como sería el caso de los llamados tratamientos preimplantacionales (Gobello 2006a; Dumon, 2009; Sánchez et al., 2010). En este sentido, cabe considerar que estudios previos destacan que solo entre el 62 y el $73 \%$ de las perras que se presentaron para interrupción de preñez se encontraban efectivamente gestantes (Feldman et al., 1993; Gobello et al., 2002).

Dado que la mantención de toda la gestación canina es completamente dependiente de la producción de progesterona $\left(\mathrm{P}_{4}\right)$ ovárica (Hoffmann et al., 2004; Concannon, 2011; Kowalewski, 2014), varias de las opciones terapéuticas descritas para interrumpir la gestación consideran el uso de fármacos como los anti-progestágenos que puedan interferir el efecto de $\mathrm{P}_{4}$ sobre el útero, o los antiprolactínicos y las prostaglandinas que actúan sobre la producción de $\mathrm{P}_{4}$ en el ovario (De La Sota et al., 2002; Fontbonne, 2010).
La aglepristona es un antagonista competitivo de $\mathrm{P}_{4}$ que se liga selectivamente al receptor, inhibiendo de este modo la cascada molecular asociada al esteroide natural (Hoffmann y Schuler, 2000). Si bien su uso como abortivo en perras se describe como eficaz y seguro desde hace varios años (Galac et al., 2000), su disponibilidad en varios países, incluyendo Chile, sigue siendo limitada. Por otra parte, agonistas dopaminérgicos y antagonistas serotoninérgicos, dado su efecto anti-prolactínico, determinan un cese en el estímulo luteotrófico de la prolactina y, por consiguiente, una caída significativa en los niveles sanguíneos de $\mathrm{P}_{4}$, ocasionando el término de la gestación cuando son administrados, preferentemente, después del día 35 de gestación (Gobello, 2006a).

Otra opción terapéutica, ampliamente descrita, es el uso de análogos sintéticos de la prostaglandina $F_{2 \dot{a}}\left(P_{2 a}\right)$ como el cloprostenol, dado que estos poseen actividad luteolítica y ecbólica; señalándose, además, como una opción eficaz y segura para la interrupción de la preñez canina a partir de la segunda mitad de gestación (Feldman et al., 1993; De La Sota et al., 2002; Wanke et al., 2002; Corrada et al., 2006; Dumon, 2009; Fontbonne, 2010). Un desafío clínico con el uso de análogos de $\mathrm{PGF}_{2 \mathrm{a}}$ es su efecto sobre la musculatura lisa, dado que generan efectos secundarios transitorios como jadeo, salivación, vómito, diarrea y taquicardia (Johnston et al., 2001; Wanke et al., 2002; Fontbonne, 2010). 
El propósito del presente reporte fue presentar la experiencia clínica de 10 casos de interrupción de gestaciones mayores o iguales de 30 días en perras de cría, utilizando un protocolo abreviado, eficaz y seguro, mediante la administración de cloprostenol cada 12 horas.

\section{Casos Clínicos}

En la Región de Valparaíso, Chile, entre los años 2013 y 2017 se recibieron para consulta de especialidad 10 perras de cría entre 2 y 5 años y de diferentes razas, todas con antecedentes de apareamiento no deseado, las cuales fueron citadas a examen clínicoreproductivo 30 días después de observada la monta por los propietarios. Las perras al momento del examen clínico presentaron parámetros fisiológicos normales.

El monitoreo ecográfico se hizo con un equipo Mindray M5Vet y transductores de 5 y 7.5 MHz, permitiendo confirmar la preñez. Mediante la medición de la cavidad coriónica interna de las vesículas gestacionales, según Becaglia y Luvoni (2012), se pudo establecer una edad gestacional máxima de 30 días (25-30 días). Una vez obtenido el consentimiento escrito de los propietarios, se procedió a aplicar el protocolo de interrupción de gestación, para cuyo diseño se consideraron dos aspectos fundamentales: la disponibilidad de fármacos en el mercado nacional y el criterio para desarrollar un tratamiento seguro y eficaz sin necesidad de hospitalización de las pacientes.

El cloprostenol utilizado, cuya presentación comercial contiene $0.25 \mathrm{mg} / \mathrm{ml}$ (Genestran ${ }^{\circledR}$, Graeub, Suiza), fue diluido con suero fisiológico para alcanzar las dosis terapéuticas en cada caso. El esquema de tratamiento consistió en la administración de 4 aplicaciones de $1 \mu \mathrm{g} / \mathrm{kg}$, vía subcutánea, cada 12 horas, seguido de 4 aplicaciones de $2 \mu \mathrm{g} /$ $\mathrm{kg}$, vía subcutánea, cada 12 horas. Las perras fueron observadas por 30 minutos des- pués de cada administración por posibles efectos colaterales (jadeo, salivación, vómito y diarrea). En todos los casos, previo a la administración de la séptima dosis de cloprostenol, se tomó una muestra de sangre de la vena cefálica con el objeto de evaluar la concentración de $\mathrm{P}_{4}$ sérica, mediante un kit comercial de ELISA semicuantitativo (Target Canine Ovulation Timing kit, BioMetallics, EEUU).

Una vez iniciado el tratamiento, se solicitó a los propietarios observar a las perras tan a menudo como fuese posible y registrar los hallazgos, considerándose como evidencia clínica de interrupción de la gestación, la presencia de flujo vaginal sanguinolento oscuro o aborto. Luego, alrededor de 12 horas después de la observación de signos de interrupción de gestación, se realizó una evaluación ecográfica para confirmar la ausencia de vesículas gestacionales en el útero. Los primeros signos de interrupción de gestación fueron reportados posteriores a la sexta o séptima administración de cloprostenol (días 3 y 4 de tratamiento) y en el examen ecográfico subsecuente se puedo constatar ausencia de vesículas gestacionales en el útero, confirmando así la eficiencia del tratamiento. En cinco $(5 / 10)$ hembras hubo aborto posterior a la séptima dosis y en las otras cinco (5/10) ocurrió reabsorción posterior a la sexta dosis, destacando el hecho de que las reabsorciones ocurrieron en las hembras con menor tiempo de gestación (25-27 días).

En el Cuadro 1 se presenta un resumen de las características de las pacientes, edad gestacional estimada al momento de inicio del tratamiento, tipo de interrupción de gestación, duración del tratamiento, niveles de progesterona y presencia de efectos colaterales en perras tratadas con cloprostenol.

Las dosis y el ritmo de adminis-tración de cloprostenol propuestos en el presente estudio resultan de una modificación del protocolo propuesto por Fieni et al. (1997), quienes describen el uso de 3 dosis de $2.5 \mu \mathrm{g} / \mathrm{kg}$, administradas vía subcutánea, cada 48 horas 
(5 días), obteniendo el 92.5\% de interrupción de gestaciones que se encontraban entre 29 y 55 días. En comparación con lo anterior, cabe destacar que el tiempo promedio de tratamiento en el presente estudio no fue superior a 4 días y que el periodo en que fueron interrumpidas las gestaciones ( $\leq 30$ días) se podría considerar relativamente temprano, 10 cual se puede contemplar como una ventaja para la práctica clínica en este tipo de situaciones y consistente con lo recomendado por Johnston et al. (2001) en el sentido que los tratamientos más precoces permiten mayor cantidad de reabsorciones que abortos.

Respecto al comportamiento de la $\mathrm{P}_{4}$ sérica, dada la confirmación de gestación y presencia de latido cardiaco en la exploración ecográfica, se puede inferir que todas las concentraciones sanguíneas, al momento de iniciar el tratamiento se encontraban sobre el valor mínimo para mantención de gestación, es decir $>5 \mathrm{ng} / \mathrm{ml}$ (Linde-Forsberg,
2010; Concannon, 2011). La concentración de $\mathrm{P}_{4}$ sérica, previo a la séptima dosis de cloprostenol, fue de $\leq 2.5 \mathrm{ng} / \mathrm{ml}$ en todas las hembras. Este resultado concuerda con Feldman et al. (1993), quienes indican que el efecto luteolítico de las prostaglandinas precede en cerca de 24 horas a la interrupción de gestación.

Otro aspecto considerado relevante para la decisión de dosis y ritmo de administración del cloprostenol estuvo relacionado a disminuir los efectos colaterales, iniciando el tratamiento con una dosis baja $(1 \mu \mathrm{g} / \mathrm{kg})$ para luego de 48 horas pasar a una dosis más alta $(2 \mu \mathrm{g} / \mathrm{kg})$, según proponen Wanke et al. (2002). En este estudio se observó la presencia de jadeo y salivación entre 5 y 10 minutos pos-inyección, mientras que el vómito se presentó entre 15 y 20 minutos más tarde en las 10 hembras. Cabe señalar que la emesis solo se presentó hasta la cuarta administración en 6 de las 10 hembras. En general, se pudo

Cuadro 1. Características de las pacientes, edad gestacional, tipo de interrupción de gestación (IG), duración del tratamiento, niveles de progesterona y presencia de efectos colaterales en 10 perras de cría tratadas con cloprostenol

\begin{tabular}{|c|c|c|c|c|c|c|c|c|}
\hline Caso & Raza & $\begin{array}{l}\text { Edad } \\
\text { (años) }\end{array}$ & $\begin{array}{r}\text { Peso } \\
(\mathrm{kg})\end{array}$ & $\begin{array}{c}\text { Edad } \\
\text { gestacional } \\
\text { (días) }\end{array}$ & Tipo de IG & $\begin{array}{c}\text { Días } \\
\text { para la } \\
\text { IG (n) }\end{array}$ & $\begin{array}{c}\text { Progesterona } \\
\text { (ng/ml) }\end{array}$ & $\begin{array}{c}\text { Efectos } \\
\text { colaterales } \\
\text { transitorios }\end{array}$ \\
\hline 1 & $\begin{array}{l}\text { Bulldog } \\
\text { Inglés }\end{array}$ & 2.5 & 18.5 & $25-27$ & Reabsorción & 6 & $1-2.5$ & $\mathrm{Si}$ \\
\hline 2 & Bulterrier & 3.0 & 18.7 & $25-27$ & Reabsorción & 6 & $1-2.5$ & $\mathrm{Si}$ \\
\hline 3 & $\begin{array}{l}\text { Yorkshire } \\
\text { Terrier }\end{array}$ & 2.0 & 02.6 & $25-27$ & Reabsorción & 6 & $1-2.5$ & $\mathrm{Si}$ \\
\hline 4 & $\begin{array}{l}\text { Bulldog } \\
\text { Francés }\end{array}$ & 4.0 & 10.5 & $25-27$ & Reabsorción & 6 & $<1$ & $\mathrm{Si}$ \\
\hline 5 & $\begin{array}{l}\text { Bulldog } \\
\text { Inglés }\end{array}$ & 4.5 & 21.6 & $25-27$ & Reabsorción & 6 & $<1$ & $\mathrm{Si}$ \\
\hline 6 & Chihuahua & 2.5 & 02.8 & $28-30$ & Aborto & 7 & $1-2.5$ & $\mathrm{Si}$ \\
\hline 7 & $\begin{array}{l}\text { Bulldog } \\
\text { Francés }\end{array}$ & 4.0 & 11.2 & $27-29$ & Aborto & 7 & $1-2.5$ & $\mathrm{Si}$ \\
\hline 8 & $\begin{array}{l}\text { Bulldog } \\
\text { Inglés }\end{array}$ & 3.0 & 19.8 & $28-30$ & Aborto & 7 & $<1$ & $\mathrm{Si}$ \\
\hline 9 & Weimaraner & 3.5 & 26.7 & $27-29$ & Aborto & 7 & $1-2.5$ & $\mathrm{Si}$ \\
\hline 10 & Shiba Inu & 5.0 & 07.2 & $27-29$ & Aborto & 7 & $1-2.5$ & $\mathrm{Si}$ \\
\hline
\end{tabular}


apreciar que los efectos secundarios fueron transitorios y disminuyeron en la medida que se avanzó en el tratamiento, lo cual concuerda con lo descrito por otros autores (Feldman et al., 1993; Fieni et al., 1997; Wanke et al., 2002; Gobello, 2006b; Sánchez et al., 2010).

Todas las hembras fueron controladas clínicamente una semana después de confirmada la interrupción de gestación, confirmándose que presentaban un buen estado de salud general. Además, luego de 4 a 6 meses volvieron a presentar un nuevo ciclo estral.

\section{Literatura Citada}

1. Beccaglia M, Luvoni G. 2012. Prediction of parturition in dogs and cats: accuracy at different gestational ages. Reprod Dom Anim. 47: 194-196. doi: 10.1111/rda.12006

2. Concannon PW. 2011. Reproductive cycles of the domestic bitch. Anim Reprod Sci 124: 200-210. doi: 10.1016/ j.anireprosci.2010.08.028

3. Corrada Y, Rodríguez R, Tortora M, Arias D, Gobello C. 2006. A combination of oral cabergoline and double cloprostenol injections to produce third-quarter gestation termination in the bitch. J Am Anim Hosp Assoc 42: 366-370. doi: 10.5326/0420366

4. De La Sota R, Soto A, Gobello C. 2002. Farmacología del estro y del parto. En: Farmacología y terapéutica veterinaria. Madrid: McGraw-Hill-Interamericana. $p$ 423-434.

5. Dumon C. 2009. Patología de la reproducción en la especie canina. Buenos Aires: Inter-Médica. $470 \mathrm{p}$.

6. Feldman EC, Davidson AP, Nelson RW, Nyland TG, Munro C. 1993. Prostaglandin induction of abortion in pregnant bitches after misalliance. J Am Vet Med Assoc 202: 1855-1858.
7. Fieni F, Dumon C, Tainturier D, Bruyas JF. 1997. Clinical protocol for pregnancy termination in bitches using prostaglandin F2 alpha. J Reprod Fertil 51:245-250.

8. Fontbonne A. 2010. Clinical approach to unwanted mating and pregnancy termination. In: BSAVA Manual of canine and feline reproduction and neonatology. $2^{\text {nd }}$ ed. Gloucester, UK: British Small Animal Veterinary Association. p 106-114.

9. Galac S, Kooistra HS, Butinar J, Bevers MM, Dieleman SJ, Voorhout G, Okkens AC. 2000. Termination of mid-gestation pregnancy in bitches with aglepristone, a progesterone receptor antagonist. Theriogenology 53: 941-950. doi: 10.1016/S0093-691X(00)00241-7

10. Gobello C, Castex G, Corrada Y, Klima L, de la Sota R, Rodríguez $R$. 2002. A comparative study of two prostaglandins and bromocriptine for pregnancy termination in bitches. J Am Vet Med Assoc 220:1017-1019.

11. Gobello C. 2006a. Control de la reproducción indeseada en la hembra. En: Wanke MM, Gobello C (eds). Reproducción en caninos y felinos domésticos. Buenos Aires: Inter-Médica. p 303-309.

12. Gobello C. 2006b. Dopamine agonists, anti-progestins, antiandrogens, longterm-release GnRH agonists and antiestrogens in canine reproduction: a review. Theriogenology 66: 1560-1567. doi: 10.1016/j.theriogenology.2006.02.-005

13. Hoffmann B, Schuler G. 2000. Receptor blockers - general aspects with respect to their use in domestic animal reproduction. Anim Reprod Sci 60-61: 295-312. doi: 10.1016/S03784320(00)00129-9

14. Hoffmann B, Büsges F, Engel E, Kowalewski M, Papa P. 2004. Regulation of corpus luteum function in the bitch. Reprod Domest Anim 39: 232-240. doi: 10.1111/j.1439-0531.2004.-00508.x 
15. Johnston S, Kustritz M, Olson P. 2001. Canine and feline theriogenology. Philadelphia, USA: WB Saunders. 592 p.

16. Kowalewski M. 2014. Luteal regression vs. prepartum luteolysis: regulatory mechanisms governing canine corpus luteum function. Reprod Biol 14: 89-102. doi: 10.1016/j.repbio.2013.11.004

17. Linde-Forsberg C. 2010. Pregnancy diagnosis, normal pregnancy and parturition in the bitch. In: England G, von Heimendahl A. BSAVA Manual of canine and feline reproduction and neonatology. $2^{\text {nd }}$ ed. Gloucester, UK: British Small Animal Veterinary Association. p 89-105.
18. Sánchez A, Rojas G, Moreno P. 2010. Interrupción de gestación en una perra mediante un tratamiento combinado de cloprostenol y cabergolina. Rev Inv Vet Perú 21: 130-135. doi: 10.15381/ rivep.v21i1.477

19. Wanke M, Romagnoli S, Verstegen J, Concannon P. 2002. Pharmacological approaches to pregnancy termination in dogs and cats including the use of prostaglandins, dopamine agonists, and dexamethasone. In: Concannon PW, England G, Verstegen J, Linde Forsberg C. (eds). Recent advances in small animal reproduction. Ithaca NY, USA: International Veterinary Information Service, (www.ivis.org) 